\title{
As Normas de Orientação Clínica em Portugal e os Valores dos Doentes
}

\author{
The Portuguese Guidelines and Patients Values
}

\author{
Paulo SANTOS $\square^{1,6}$, Isabel NAZARÉ2 ${ }^{\text {, Carlos MARTINS }}{ }^{1,6}$, Luísa SÁ ${ }^{1,3}$, Luciana COUTO ${ }^{1,4}$, Alberto HESPANHOL HE $^{1,5,6}$ \\ Acta Med Port 2015 Nov-Dec;28(6):754-759
}

\section{RESUMO}

Introdução: As normas de orientação clínica são instrumentos de apoio à decisão que visam a melhoria da qualidade, promovendo as boas práticas clínicas. Os cuidados orientados para o utente permitem uma melhor satisfação e autogestão da saúde, com ganhos de qualidade e potencialmente menores custos.

Objetivo: Avaliar o grau de integração dos valores dos doentes nas normas de orientação clínica publicadas em Portugal.

Material e Métodos: Reviram-se as 18 normas de orientação clínica da área cardiovascular publicadas em Portugal entre 2011 e 2013, procurando avaliar a introdução das ideias, medos, expectativas e preferências dos doentes.

Resultados: Oito normas de orientação clínica estavam relacionadas com processos de diagnóstico e 10 com aspetos de terapêutica. Em cinco normas de orientação clínica (28\%) foram encontradas referências a valores dos doentes, todas na área da terapêutica, não existindo nenhuma nas normas de orientação clínica relacionadas com o diagnóstico. A incorporação das expectativas dos doentes foi o aspeto mais presente. Em $78 \%$ existiam referências à valorização dos custos financeiros.

Discussão: As normas de orientação clínica constituem-se como uma tecnologia de saúde ao dispor dos profissionais com o objetivo de melhorar a prestação dos cuidados assistenciais aos cidadãos, utilizadores últimos destes instrumentos. Como noutros países, há uma tendência a não considerar os utentes e os seus sistemas de valores na estrutura das orientações, privilegiando-se uma lógica populacional nem sempre aplicável ao caso concreto, e uma aritmética financeira pouco suportada em avaliações de custoefetividade. No caso português, a forma como foram propostas foi também condicionadora de desconfiança tanto nos profissionais como nos utentes.

Conclusão: As normas de orientação clínica em Portugal apresentam uma baixa taxa de incorporação dos valores dos utentes, de uma forma mais visível na definição do diagnóstico, o que os coloca numa posição secundária no processo de decisão clínica com potenciais perdas na qualidade.

Palavras-chave: Normas de Orientação Clínica; Portugal; Preferências dos Doentes; Qualidade de Cuidados de Saúde; Relação Médico-Doente.

\section{ABSTRACT}

Introduction: Clinical guidelines are support tools, aiming to improve quality of the clinical practice. Patient centered care allows best satisfaction rates, with greater health self-management, and potential gains in quality with fewer costs.

Objective: To evaluate the integration of patients' values in Portuguese guidelines.

Material and Methods: We reviewed the 18 guidelines about cardiovascular diseases published in Portugal from 2011 to 2013 , searching for the integration of patients' ideas, fears, expectations and preferences.

Results: Eight guidelines were related to diagnosis approach and 10 with treatment. We found references to patients' values in 5 (28\%) guidelines, all about treatment aspects. The incorporation of patients' expectations was the most present feature. Reference to financial costs was present in $78 \%$ of the guidelines.

Discussion: Clinical guidelines are health technology instruments available to practitioners in order to improve the quality of care provided to patients, who are the real users of these tools. As in other countries, there is a tendency to disregard the users' value systems in the conception of the guidelines, giving the privilege to a population logic, not always applicable to the individual, and to financial arithmetic with low support in cost-effectiveness assessments. In the Portuguese case, the way guidelines were proposed conditioned also some suspicion both in the professionals and in the users.

Conclusion: Portuguese guidelines have low incorporation of references to patients' values. This is more evident when questions about diagnosis are addressed, placing patients in a secondary role in the clinical decision-making process with potential losses in quality of care and eventual increase in costs.

Keywords: Guideline; Patient Preference; Physician-Patient Relations; Portugal; Quality of Health Care.

\section{INTRODUÇÃO}

Na definição original de 1990, guideline é um documento desenvolvido de forma sistematizada para auxiliar o médico e o paciente nas decisões sobre os melhores cuidados de saúde para uma situação específica, ${ }^{1}$ destinando-se a implementar um sistema de melhoria contínua da qualidade, adequação e efetividade dos serviços de saúde,

1. Unidade de Medicina Geral e Familiar. Departamento de Ciências Sociais e Saúde. Faculdade de Medicina. Universidade do Porto. Porto. Portugal.

2. Unidade de Cuidados de Saúde Personalizados de Avintes. Vila Nova de Gaia. Portugal.

3. Unidade de Saúde Familiar Nova Via. Valadares. Portugal.

4. Unidade de Saúde Familiar Camélias. Vila Nova de Gaia. Portugal.

5. Unidade de Saúde Familiar S. João do Porto. Porto. Portugal.

6. Centro de Investigação em Tecnologias e Serviços de Saúde (CINTESIS). Universidade do Porto. Porto. Portugal.

$\triangle$ Autor correspondente: Paulo Santos. psantosdr@med.up.pt

Recebido: 09 de Fevereiro de 2015 - Aceite: 27 de Julho de 2015 | Copyright @ Ordem dos Médicos 2015 
promovendo as boas práticas clínicas. ${ }^{2}$

Neste sentido, a existência de diretrizes cientificamente robustas que orientem o exercício clínico pode ser considerada uma estratégia de equidade que impulsiona tanto os profissionais de saúde como os cidadãos utilizadores dos seus serviços a uma prática com maior transparência baseada na melhor evidência disponível, afastando definitivamente os fantasmas da medicina paternalista em que a decisão médica paradigmática se revestia de um poder absoluto.

A aplicação desta medicina baseada na evidência afirma o primado da justiça enquanto princípio ético basilar, propondo soluções eficientes para o caso concreto e para os sistemas de saúde, sem comprometer a beneficência que o médico deve ao seu paciente, na medida em que incorpora a experiência clínica e os valores dos doentes nos dados da investigação ${ }^{3}$ e no processo de decisão.

Trata-se de uma medicina orientada para os doentes, mais do que orientada para as doenças, que poderá trazer mais e melhores ganhos em saúde, ${ }^{4}$ o que é de todos um objetivo.

Um pouco por todo o mundo, agências especializadas publicam com regularidade orientações clínicas. Em Portugal, foi legalmente atribuída à Direção Geral da Saúde (DGS) a função de emitir normas e orientações tanto clínicas como organizativas. ${ }^{5}$ Em 2011, a necessidade de assinar um acordo internacional de assistência financeira, le- vou o Estado Português a comprometer-se com uma reorganização económica e social a vários níveis. ${ }^{6}$ No sector da saúde um dos objetivos passou pela publicação de normas de orientação clínica (NOC's) e pela criação de um sistema de auditoria à sua implementação.

Foram então publicadas 70 NOC's em 2011, 39 em 2012, e 32 em 2013, envolvendo áreas clínicas dos Cuidados de Saúde Primários, Hospitalares e de Reabilitação, numa perspetiva de promover a melhoria contínua de cuidados de saúde em Portugal, apesar de apenas 16 terem sido validadas no acordo estabelecido com a Ordem dos Médicos.

O objetivo deste trabalho é analisar o nível de incorporação dos valores dos doentes nas NOC's publicadas em Portugal nos anos de 2011-13.

\section{MATERIAL E MÉTODOS}

Realizou-se uma revisão das NOC's publicadas pela DGS em Portugal entre 2011 e 2013, conforme os textos disponíveis no sítio eletrónico em www.dgs.pt, consultado em 01/02/2014. (Tabela 1).

As doenças cardiovasculares são a principal causa de morte em Portugal e ocupam uma parte significativa dos recursos disponíveis, representando uma prioridade em saúde. ${ }^{7}$ Por isso selecionaram-se as NOC's relativas a patologia cardiovascular em adultos, num total de 18 das 141 disponíveis. Foram publicadas atualizações à versão

Tabela 1 - Normas de orientação clínica incluídas no estudo

\begin{tabular}{|c|c|c|c|}
\hline ID & Título & Referência & Data \\
\hline 1 & Abordagem terapêutica da hipertensão arterial & $026 / 2011$ & $19-03-2013$ \\
\hline 2 & Abordagem terapêutica das dislipidemias & 019/2011 & $11-07-2013$ \\
\hline 3 & Abordagem terapêutica farmacológica da angina estável & 046/2011 & $27-11-2013$ \\
\hline 4 & Abordagem terapêutica farmacológica na diabetes mellitus tipo 2 & $052 / 2011$ & $30-07-2013$ \\
\hline 5 & Avaliação do risco cardiovascular SCORE & $005 / 2013$ & $26-11-2013$ \\
\hline 6 & Diagnóstico e classificação da diabetes mellitus & $002 / 2011$ & $14-01-2011$ \\
\hline 7 & Ecodoppler cerebrovascular & $031 / 2011$ & $30-09-2011$ \\
\hline 8 & Ecodoppler venoso dos membros inferiores & 030/2011 & $30-09-2011$ \\
\hline 9 & Hipertensão arterial: definição e classificação & 020/2011 & $19-03-2013$ \\
\hline 10 & Insulinoterapia na diabetes mellitus tipo 2 & 025/2011 & $30-07-2013$ \\
\hline 11 & Prescrição de exames laboratoriais para avaliação de dislipidemias & 066/2011 & $30-05-2013$ \\
\hline 12 & Acidente vascular cerebral: prescrição de Medicina Física e de Reabilitação & $054 / 2011$ & $27-12-2011$ \\
\hline 13 & Prescrição e determinação da hemoglobina glicada A1c & 033/2011 & $06-12-2012$ \\
\hline 14 & Revascularização miocárdica: acompanhamento hospitalar e em cuidados de saúde primários & $037 / 2012$ & $30-12-2012$ \\
\hline 15 & Terapêutica da diabetes mellitus tipo 2: metformina & $001 / 2011$ & 07-01-2011 \\
\hline 16 & Tratamento conservador médico da insuficiência renal crónica estádio 5 & $017 / 2011$ & $14-06-2012$ \\
\hline 17 & Utilização de ecodoppler arterial dos membros inferiores & 034/2011 & $30-09-2011$ \\
\hline 18 & Utilização e seleção de antiagregantes plaquetários em doenças cardiovasculares & $014 / 2011$ & 08-07-2013 \\
\hline
\end{tabular}

Fonte: Sítio eletrónico da Direção Geral da Saúde. www.dgs.pt. Consultado 2014 fev 01. 
original em 10 NOC's. Para efeitos desta revisão apenas se considerou a última versão disponível, conforme publicada no sítio eletrónico da DGS.

\section{Recolha de dados}

Dois autores reviram os textos das NOC's, de forma independente, incluindo os anexos, para avaliação do grau de incorporação dos valores dos doentes, traduzido pelas suas ideias, preocupações, expectativas, satisfação e participação no processo de decisão médica. Na análise foi considerado o resultado final, obtido por consenso após confronto dos dados obtidos nas leituras individuais.

\section{Variáveis estudadas}

Para a sistematização da pesquisa procedeu-se à definição prévia das variáveis a estudar. Ideias foram definidas como as opiniões dos doentes sobre um possível diagnóstico, tratamento ou prognóstico. ${ }^{8}$ Por preocupações entendeu-se a expressão de receio ou medo de um diagnóstico ou tratamento. ${ }^{8} \mathrm{~A}$ expectativa dos doentes foi definida como a expressão de uma expectação sobre o diagnóstico, tratamento ou um procedimento médico. ${ }^{8}$ Por satisfação entendeu-se o grau em que o doente perceciona a utilidade, efetividade ou benefício do serviço de saúde ou tecnologia. A participação da decisão clínica foi definida pela expressão da inclusão da vontade do doente na decisão diagnóstica ou terapêutica. ${ }^{9}$

Foram estudadas também a presença de referências à avaliação da adesão do doente a medidas diagnósticas ou terapêuticas, expressão de custos financeiros, quer para o doente quer para o sistema de saúde, expressão de custos iatrogénicos, relativos a potenciais efeitos adversos dos procedimentos propostos, e a presença de indicadores de avaliação. Os indicadores foram categorizados em clínicos se pretendiam avaliar objetivos de natureza clínica, como por exemplo na NOC 1, a avaliação da proporção de inscritos com diagnóstico de hipertensão, com história de acidente vascular cerebral ou enfarte agudo do miocárdio ocorrido nos últimos 12 meses, ou não clínicos para os restantes, como por exemplo na mesma NOC a avaliação do custo da prescrição dos medicamentos antihipertensores por doente hipertenso. As NOC's foram categorizadas de acordo com o seu conteúdo no grupo das que estavam relacionadas com processos de diagnóstico da situação clínica e no grupo das que se debruçavam sobre os aspetos de terapêutica.

A variável expressão de custos financeiros foi dicotomizada, contendo qualquer referência a aspetos relacionados com custos, independentemente de estarem integrados numa análise económica ou se referirem meramente a preços de mercado ou volume de prescrição.

\section{RESULTADOS}

Foram revistas 18 NOC's contendo 88731 palavras distribuídas por 240 páginas. Dez NOC's abordavam aspetos relativos à terapêutica e oito eram respeitantes a questões relacionadas com o diagnóstico. (Fig. 1)

A incorporação dos valores dos doentes estava presente em cinco NOC's, todas na área de aspetos terapêuticos. A Fig. 1 mostra a distribuição dos resultados em função da classificação em termos do objeto da norma ser direcionado para o diagnóstico ou para questões terapêuticas.

A referência a ideias e opiniões dos doentes estavam presentes em três NOC's, através de expressões como 'A opção por qualquer dos fármacos depende...da preferência do doente' (NOC 3 da Tabela 1), 'A eficácia terapêutica ... depende ... da aceitação da terapêutica' (NOC 10) e 'adequado esclarecimento do doente e dos seus próximos acerca dos resultados esperados e contribuir, desta forma, para uma opção informada por uma modalidade de tratamento, mais ou menos agressiva, de acordo com a sua perspetiva pessoal' (NOC 16).

Expressão de ideias, preocupações e expectativas

Avaliação da adesão

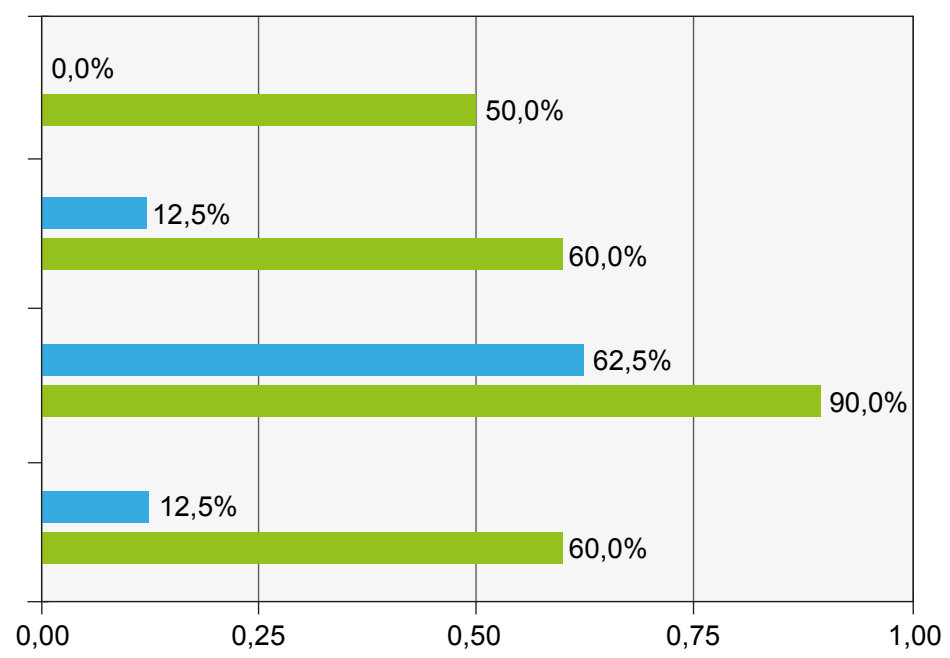

Expressão de custos financeiros

Expressão de custos iatrogénicos

NOC's relativas a diagnóstico

NOC's relativas a terapêutica

Figura 1 - Resultados da avaliação das normas de orientação clínica na área cardiovascular quanto às variáveis estudadas. NOC's: Normas de orientação clínica 
Em duas NOC's havia referência à expressão de medos ou preocupações dos doentes, traduzidas por 'a eficácia terapêutica ... depende ... da desmontagem dos falsos mitos e receios' (NOC 10) e 'em alguns momentos da sua vida futura, podem surgir ansiedade, receio do futuro, depressão' (NOC 16).

A expectativa dos doentes era abordada em duas NOC's: 'A eficácia terapêutica ... depende ... da aceitação da terapêutica' (NOC 10) e 'considerar, para cada doente, o direito à escolha do melhor tratamento para a sua pessoa, no seu contexto individual e familiar, de acordo com as suas expectativas e opções de vida' (NOC 16).

O processo de decisão partilhada na gestão dos cuidados de saúde estava presente em quatro NOC's, relevando a capacitação do doente (NOC 10), o seu envolvimento e da sua família na decisão (NOC 12), e a possibilidade de livremente fazer a sua opção informada (NOC's 1 e 16), prevendo-se mecanismos de avaliação formal ou informal da satisfação dos doentes em três NOC's.

\section{DISCUSSÃO}

Os valores dos doentes estão pouco representados nas normas de orientação clínica da área das doenças cardiovasculares publicadas em Portugal de 2011 a 2013, onde três em cada quatro NOC's não apresentam qualquer tipo de proposta relacionada com a incorporação das ideias, receios, expectativas e partilha de decisão terapêutica ou diagnóstica com os doentes. Mesmo quando presentes, as expressões resumem-se a pequenas frases inseridas no contexto da decisão médica sobre a melhor opção para o doente e não tanto no espírito de uma partilha efetiva de responsabilidade nas soluções disponíveis.

Este problema é comum noutras realidades. No Canadá, McCormack ${ }^{10}$ analisa cinco guidelines na área terapêutica, em 2007, e encontra resultados semelhantes, e Chong ${ }^{11}$ conclui pela fraca integração das preferências dos doentes. Nos Estados Unidos, MacLean et al, ${ }^{12}$ num consenso sobre terapêutica antitrombótica, chama a atenção para a necessidade de integrar as opiniões dos doentes na decisão clínica como estratégia para melhorar os resultados da intervenção médica.

Em Portugal, a escassa integração dos valores dos doentes nas orientações clínicas assume particular preocupação, se se pensar que as NOC's são apresentadas com um carácter normativo, o que não acontece noutros países.

$\mathrm{Na}$ visão biomédica clássica, o doente é portador de uma constelação de sinais e sintomas que orientam para uma lista de diagnósticos diferenciais de onde emana a doença que o define e para a qual é estabelecido um plano terapêutico e um prognóstico. A introdução do conceito de cuidados centrados no doente ${ }^{13}$ veio alterar este paradigma, inserindo no ato médico uma visão holística da saúde, relevando o papel da pessoa com doença na gestão do seu próprio processo e integrando a sua multidimensionalidade biológica, psicológica, social e espiritual nas estratégias de diagnóstico, terapêutica e prognóstico. O papel do profissional de saúde aparece como aliado ativo nes- te percurso e não como condutor único, admitindo os conhecimentos e experiências dos doentes na decisão ${ }^{14}$ que assim passa a ser partilhada por ambos, ${ }^{15}$ sem com isto diminuir a relevância do médico enquanto pessoa ${ }^{16}$ e a sua responsabilidade no manuseamento das tecnologias disponíveis.

No modelo proposto por Mead e Bower ${ }^{13}$ em 2000 eram identificados múltiplos fatores que de alguma forma podiam interferir com a obtenção deste equilíbrio e que dependiam do médico, do doente, do contexto do exercício, de variáveis socioculturais e da própria consulta, sugerindo a necessidade de uma relação continuada e estável de conhecimento mútuo para os ultrapassar.

Neste trabalho estudamos uma variável que tem a ver com o contexto do exercício ao propor um articulado normativo condicionador da habitual atividade profissional. As NOC's surgiram nos anos estudados a um ritmo muito rápido, que dificultou a apropriação dos conteúdos pelos profissionais, criando desconfiança nos profissionais quanto à fundamentação científica e originando dúvidas sobre a sua aplicabilidade ao caso concreto. ${ }^{17} \mathrm{Na}$ realidade, a simples publicação de normas não é fator suficiente para promover uma rápida mudança de comportamentos,$^{18}$ mas predispõe a uma alteração de atitudes dos profissionais que pode e deve ser aproveitada para o início de um processo de meIhoria de qualidade que ciclicamente e em continuidade venha a melhorar a situação atual.

Torna-se evidente a necessidade de orientações baseadas na melhor evidência existente ${ }^{1}$ e não apenas no consenso de peritos, adaptáveis à realidade local e à decisão personalizada e que incluam a valorização da experiência clínica e das expectativas das pessoas a quem se destinam.

Nas NOC's avaliadas, a expressão de custos financeiros assume uma relevância marcada ao estar presente na maioria dos textos. Porém não se pode admitir que reflita uma verdadeira análise económica, que obedece a uma metodologia própria e estes estudos, se existentes para a realidade nacional, não foram incluídos no articulado das NOCs. Na realidade, o financiamento da Saúde em Portugal é distribuído pelos setores público e privado, com um peso médio no sector público na ordem dos $68,5 \%$ nos anos de 2001 a 2010, segundo os dados da OCDE, com tendência a decrescer fixando-se nos $62,6 \%$ na estimativa para 2012, e representando um investimento nacional que se situou nos $10,2 \%$ do produto interno bruto em 2009 e 2010. No entanto quando a avaliação é feita em termos monetários per capita, verificamos que o custo da saúde em Portugal é sistematicamente inferior à média dos países da OCDE, com uma diferença que se tem vindo a acentuar ao longo dos últimos anos (Fig. 2), e que em 2011 se situou em -750 US\$, para níveis de saúde globais avaliados pela esperança média de vida e pela taxa de mortalidade infantil superiores ao comparador.

Proporcionar aos cidadãos os melhores cuidados de saúde disponíveis é um imperativo ético que a todos obriga. O princípio da justiça, no contexto de uma situação em 


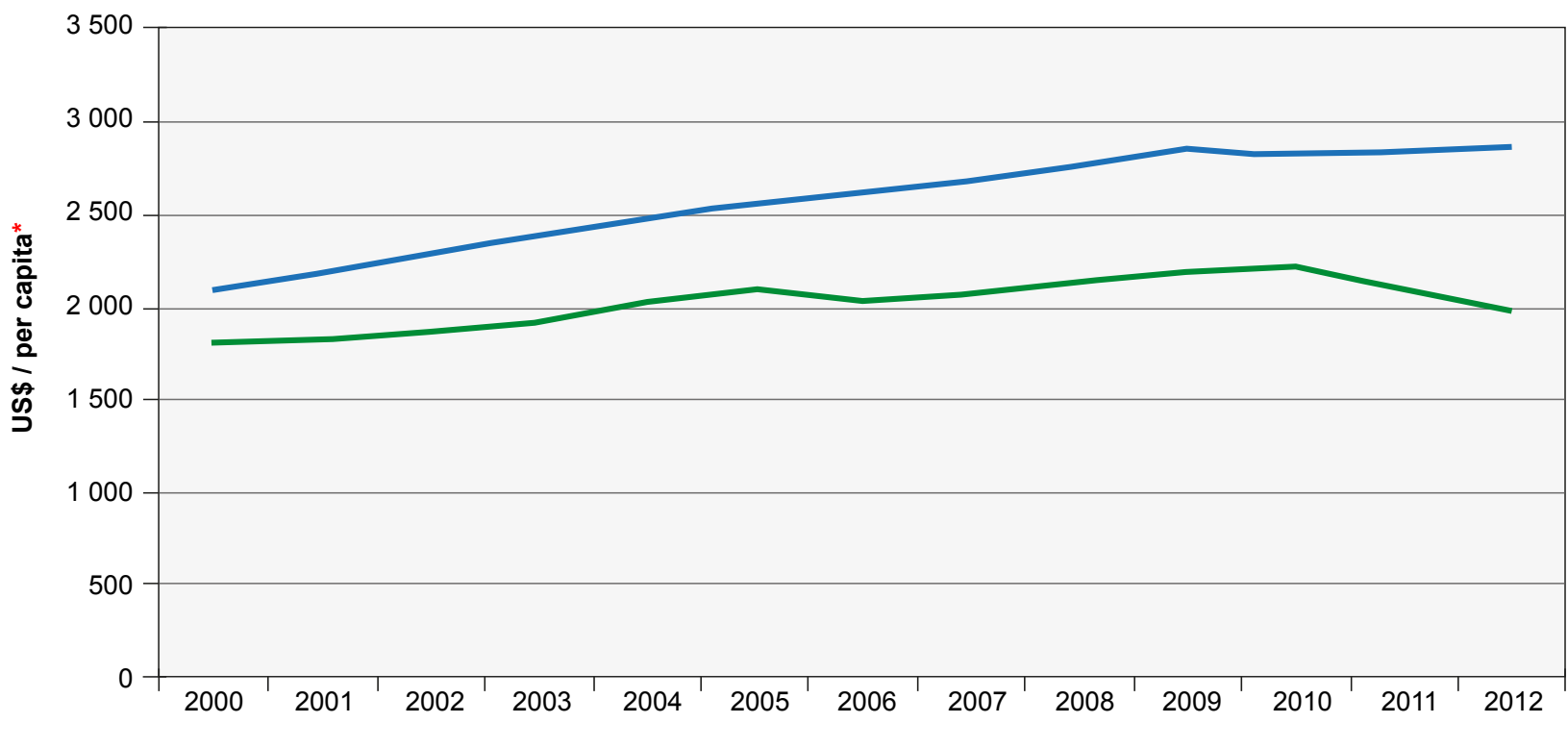

Ano

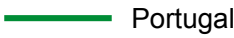

OCDE

Figura 2 - Evolução dos custos em saúde na situação portuguesa por comparação com a média da OCDE.

* Unidade monetária: Dólares norte-americanos, em paridade do poder de compra, base 2005. (Fonte: OECD Health Data, 2014)

que os recursos são limitados, leva à necessidade de uma ponderação racional da alocação dos meios disponíveis, conciliando a beneficência basilar do exercício médico com os constrangimentos resultantes de uma conjuntura adversa. As NOC's podem aqui contribuir positivamente para este aparente dilema ético, ao apoiar a decisão clínica, revendo a melhor evidência existente quanto a questões-chave para os serviços de saúde e as suas consequências e propondo-as num formato de compreensão simples que permita a sua aplicabilidade no caso concreto, ${ }^{19}$ assumindo uma lógica centrada no doente ${ }^{20}$ em vez de centrada na população ou nos serviços de saúde. Este aspeto poderá ser melhorado se incorporarmos a evidência das preferências dos doentes nos fluxogramas de decisão. ${ }^{21,22}$

Um problema que encontramos neste estudo é a falta de escalas uniformizadas e validadas de avaliação dos valores dos doentes, o que implica um risco de subjetividade na avaliação. ${ }^{23}$ Selecionamos da literatura as definições das variáveis em estudo e aceitamo-las por consenso no sentido de minimizar este risco, procurando que os resultados traduzam a realidade das NOC's publicadas entre 2011 e 2013.

\section{CONCLUSÃO}

Muito se tem falado e escrito sobre as Normas de Orientação Clínica que têm vindo à discussão pública. Avaliamos nesta revisão as NOC's da área cardiovascular publicadas em Portugal no que respeita à incorporação dos valores dos doentes, traduzida pelas suas ideias, preocupações e expectativas em relação à sua saúde e à forma como é orientada com o seu médico, concluindo pelo seu baixo grau de integração nos textos.

A introdução de orientações clínicas é positiva no sentido de facilitar a revisão da vasta evidência existente e deve ser vista numa lógica de melhoria contínua da qualidade. A eficácia deste instrumento depende de fatores relacionados com os profissionais, com os doentes e com o ambiente que os integra, ${ }^{24}$ implicando na sua conceção um olhar mais atento para as características dos doentes ${ }^{25}$ que são em última análise os reais utilizadores desta tecnologia.

\section{PROTECÇÃO DE PESSOAS E ANIMAIS}

Os autores declaram que os procedimentos seguidos estavam de acordo com os regulamentos estabelecidos pelos responsáveis da Comissão de Investigação Clínica e Ética e de acordo com a Declaração de Helsínquia da Associação Médica Mundial.

\section{CONFIDENCIALIDADE DOS DADOS}

Os autores declaram ter seguido os protocolos do seu centro de trabalho acerca da publicação dos dados de doentes.

\section{CONFLITO DE INTERESSES}

Os autores declaram não ter nenhum conflito de interesses relativamente ao presente artigo.

\section{FONTES DE FINANCIAMENTO}

Não existiram fontes externas de financiamento para a realização deste artigo. 


\section{REFERÊNCIAS}

1. Field MJ, Lohr KN, Institute of Medicine (US). Committee to Advise the Public Health Service on Clinical Practice Guidelines, United States. Department of Health and Human Services. Clinical practice guidelines: directions for a new program. Washington: National Academy Press; 1990. p. 160.

2. Recommendation $\operatorname{Rec}(2000) 3$ : Developing a methodology for drawing up guidelines on best medical practices. Brussels: Committee of Ministers of the Council of Europe: Council of Europe Publishing; 2001.

3. Sackett DL. Evidence-based medicine: how to practice and teach EBM. $2^{\text {nd }}$ ed. Edinburgh, New York: Churchill Livingstone; 2000.

4. Rathert C, Wyrwich MD, Boren SA. Patient-centered care and outcomes: a systematic review of the literature. Med Care Res Rev. 2013;70:35179.

5. Portugal, Ministério da Saúde. Decreto Regulamentar n. ${ }^{\circ} 14 / 2012$ de 26 de janeiro. Diário da República, $1 .^{a}$ série - N. ${ }^{\circ} 19.2012 ; 480-2$.

6. Portugal: Request for a Three-Year Arrangement Under the Extended Fund Facility, IMF Country Report No. 11/127, June 2011. | Council Implementing Decision on Granting Financial Assistance to Portugal, 17 May 2011. Lisboa: Banco de Portugal; 2011.

7. Bloom DE. The health and wealth of Portugal. Acta Med Port. 2013;26:303-11.

8. Matthys J, Elwyn G, Van Nuland M, Van Maele G, De Sutter A, De Meyere M, et al. Patients' ideas, concerns, and expectations (ICE) in general practice: impact on prescribing. Br J Gen Pract. 2009;59:29-36.

9. Kon AA. The shared decision-making continuum. JAMA. 2010;304:9034.

10. McCormack JP, Loewen P. Adding "value" to clinical practice guidelines. Can Fam Physician. 2007;53:1326-7.

11. Chong CA, Chen IJ, Naglie G, Krahn MD. How well do guidelines incorporate evidence on patient preferences? J Gen Intern Med. 2009;24:977-82

12. MacLean S, Mulla S, Akl EA, Jankowski M, Vandvik PO, Ebrahim S, et al. Patient values and preferences in decision making for antithrombotic therapy: a systematic review: Antithrombotic Therapy and Prevention of Thrombosis. $9^{\text {th }}$ ed. American College of Chest Physicians EvidenceBased Clinical Practice Guidelines. Chest. 2012;141:e1S-23.
13. Mead N, Bower P. Patient-centredness: a conceptual framework and review of the empirical literature. Soc Sci Med. 2000;51:1087-110.

14. Byrne PS, Long BE. Doctors talking to patients. a study of the verbal behaviour of general practitioners consulting in their surgeries. London: Her Majesty's Stationery Office; 1976.

15. Charles C, Gafni A, Whelan T. Decision-making in the physician-patient encounter: revisiting the shared treatment decision-making model. Soc Sci Med. 1999;49:651-61.

16. Balint E. The doctor, the patient, and the group: Balint revisited. New York: Routledge; 1993.

17. Granja M. As normas da DGS: tempo de oportunidade e responsabilidade para os médicos de família. Rev Port Clín Geral. 2011;27:417-8.

18. Lomas J, Anderson GM, Domnick-Pierre K, Vayda E, Enkin MW, Hannah WJ. Do practice guidelines guide practice? The effect of a consensus statement on the practice of physicians. N Engl J Med. 1989;321:130611.

19. Jackson $R$, Feder $G$. Guidelines for clinical guidelines. BMJ. 1998;317:427-8.

20. Rumsfeld D. Guiding the guidelines. Lancet. 2011;377:1125.

21. Little P, Everitt $H$, Williamson I, Warner G, Moore M, Gould C, et al. Observational study of effect of patient centredness and positive approach on outcomes of general practice consultations. BMJ. 2001;323:908-11.

22. Stewart M, Brown JB, Donner A, McWhinney IR, Oates J, Weston WW, et al. The impact of patient-centered care on outcomes. J Fam Pract. 2000;49:796-804.

23. Hudon C, Fortin M, Haggerty JL, Lambert M, Poitras ME. Measuring patients' perceptions of patient-centered care: a systematic review of tools for family medicine. An Fam Med. 2011;9:155-64.

24. Francke AL, Smit MC, de Veer AJ, Mistiaen P. Factors influencing the implementation of clinical guidelines for health care professionals: a systematic meta-review. BMC Med Inform Decis Mak. 2008;8:38.

25. Santos P, Martins C, Sá L, Hespanhol A, Couto L. Motives for requesting an electrocardiogram in primary health care. Ciência Saúde Coletiva. 2015;20:1549-54 
Paulo SANTOS, Isabel NAZARÉ, Carlos MARTINS, Luísa SÁ, Luciana COUTO, Alberto HESPANHOL

\section{As Normas de Orientação Clínica em Portugal e os Valores dos Doentes}

Acta Med Port 2015:28:754-759

Publicado pela Acta Médica Portuguesa, a Revista Científica da Ordem dos Médicos

Av. Almirante Gago Coutinho, 151

1749-084 Lisboa, Portugal.

Tel: +351218428215

E-mail: submissao@actamedicaportuguesa.com

www.actamedicaportuguesa.com

ISSN:0870-399X | e-ISSN: 1646-0758

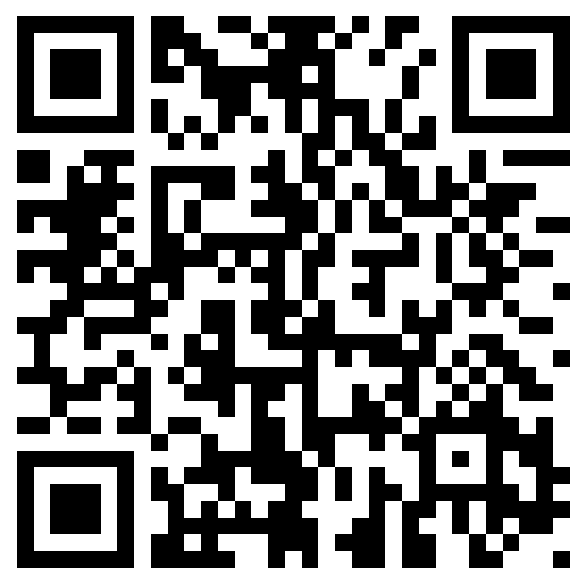

ACTA MÉDICA

PORTUGUESA 